\title{
Temperature and Heat Flux Estimation from Sampled Transient Sensor Measurements
}

\author{
Z. C. Feng ${ }^{1}$, J. K. Chen ${ }^{2}$, Yuwen Zhang ${ }^{3}$ \\ Department of Mechanical and Aerospace Engineering \\ and \\ Stephen Montgomery-Smith ${ }^{4}$ \\ Department of Mathematics \\ University of Missouri \\ Columbia, MO 65211, USA
}

\begin{abstract}
Laplace transform is used to solve the problem of heat conduction over a finite slab. The temperature and heat flux on the two surfaces of a slab are related by the transfer functions. These relationships can be used to calculate the front surface heat input (temperature and heat flux) from the back surface measurements (temperature and/or heat flux) when the front surface measurements are not feasible to obtain. This paper demonstrates that the front surface inputs can be obtained from the sensor data without resorting to inverse Laplace transform. Through Hadamard Factorization Theorem, the transfer functions are represented as infinite products of simple polynomials. Consequently, the relationships between the front and back surfaces are translated to the time-domain without inverse Laplace transforms. These timedomain relationships are used to obtain approximate solutions through iterative procedures. We select a numerical method that can smooth the data to filter out noise and at the same time obtain the time derivatives of the data. The smoothed data and time derivatives are then used to calculate the front surface inputs.
\end{abstract}

\footnotetext{
${ }^{1}$ Corresponding author. Email: fengf@missouri.edu, Fax: 573-884-5090, Phone: 573-884-4624.

${ }^{2}$ Email: chenjnk@missouri.edu

${ }^{3}$ Email: zhangyu@missouri.edu

${ }^{4}$ Email: montgomerysmith@missouri.edu
} 


\section{NOMENCLATURE}

$\begin{array}{ll}c_{p s} & \text { mass specific heat of the slab, } \mathrm{J} /(\mathrm{kg} \cdot \mathrm{K}) \\ G & \text { Transfer function } \\ i & \sqrt{-1} \\ k_{s} & \text { thermal conductivity, } \mathrm{W} /(\mathrm{m} \cdot \mathrm{K}) \\ L & \text { thickness of 1-D slab, } \mathrm{m} \\ p & \text { pole of a transfer function } \\ q & \text { heat flux, W/m }{ }^{2} \\ Q & \text { Laplace transform of the heat flux } \\ t & \text { time, s } \\ t_{c} & \text { characteristic time, s } \\ T_{s} & \text { temperature of the slab above the ambient temperature, } \mathrm{K} \\ U & \text { Laplace transform of temperature } \\ x & \text { spatial coordinate variable, m } \\ z & \text { zero of a transfer function }\end{array}$

\section{Greek symbols}

$\begin{array}{ll}\rho_{s} & \text { density of the slab, } \mathrm{kg} / \mathrm{m}^{3} \\ \tau & \text { dimensionless time } \\ \omega & \text { dimensionless frequency } \\ \xi & \text { dimensionless length variable }\end{array}$

\section{Subscripts}

b back surface quantity

front surface quantity

Keywords: Inverse problem, transfer function, Savitzky-Golay, sensor compensation, temperature measurement 


\section{Introduction}

To conduct thermal measurements under harsh environment, it has been proposed that sensors be located away from direct contact with the environment. The desired measurements are obtained by considering the heat transfer paths between the points of interest and the sensors. Imagine a thin plate separating the environment and the sensors that are mounted on the back surface. The front surface temperature can be determined indirectly by solving an inverse heat conduction problem [1-13] based on the transient temperature and/or heat flux measured at the back surface. Among the many methods proposed to solve the inverse heat conduction problem, the Laplace transform method (if applicable) most concisely captures the mathematical relationships in terms of transfer functions [14-18]. To obtain the desired quantities from the sensor measurements, the inverse Laplace transform must be found. Since closed form inverse Laplace transform rarely exists for the heat equations, many algorithms for the approximate inverse Laplace transform have been proposed for this purpose [18-24].

Most of the approximate inverse Laplace transform algorithms start from known functions in the Laplace domain. This presents a severe restriction since the time-domain sensor data must be transformed into the Laplace domain. Obtaining meaningful Laplace transform from the sensor data is no trivial matter considering the unavoidable noise contamination. Moreover, most sensor data are discretized data sampled at equal time intervals. The nature of the sampled data adds to the complexity of reconstructing the inverse Laplace transform.

In our previous work, we have proposed polynomial approximations to the transfer functions [18]. In this paper, we use the Hadamard Factorization Theorem to express transfer functions as infinite products. Through the infinite product expansion of the transfer functions, time domain relationships between the front and back surfaces are obtained. These relationships are implemented in iterative procedures.

In the following section, we present the simplified model for the heat equation and the transfer functions relating the thermal quantities between the front and back surfaces. In section 3, iterative procedures are developed. In section 4, we present the results of reconstructed front surface heat flux and temperature by using the back surface temperature from the analytical solution as the measurement data. Noise is added to the analytical data. A conclusion is given in section 5.

\section{Mathematical model and the Laplace transform solution}

Consider one-dimensional heat conduction over a finite slab. The governing equation is given by the following: 


$$
\rho_{s} c_{p s} \frac{\partial T_{s}}{\partial t}=k_{s} \frac{\partial^{2} T_{s}}{\partial x^{2}}
$$

where $\rho_{s}, k_{s}$ and $c_{p s}$ are the density, thermal conductivity and specific heat of the solid; they are all assumed to be constant.

Since the one dimensional model here represents an approximation of a sheet-like threedimensional body when the temperature gradients in the in-plane directions are ignored, it is thus more intuitive to regard $x=0$ and $x=L$ as the "front" and the "back" surfaces of the sheet-like solid. To simplify the problem further, we choose the sheet thickness $L$ as the characteristic length and the constant

$$
t_{c}=\frac{\rho_{s} c_{p s} L^{2}}{k_{s}}
$$

as the characteristic time. Thus we simplify the governing equation to

$$
\frac{\partial T_{s}}{\partial \tau}=\frac{\partial^{2} T_{s}}{\partial \xi^{2}} \quad \text { for } 0<\xi<1
$$

where $\tau=t / t_{c}$ and $\xi=x / L$ now represent the dimensionless time and dimensionless position across the thickness.

The boundary conditions can be prescribed temperatures on the front and back surfaces $T_{f}(t)$ and $T_{b}(t)$. Or the heat fluxes on the front surface and back surface can be prescribed, i.e.

$$
\begin{aligned}
& -\frac{\partial T_{s}}{\partial \xi}(\xi=0)=q_{f}(t) \\
& -\frac{\partial T_{s}}{\partial \xi}(\xi=1)=q_{b}(t)
\end{aligned}
$$

where $q_{f}(t)$ and $q_{b}(t)$ are normalized heat flux $(\mathrm{K})$ at the front and back surfaces. The actual front surface heat flux is $q_{f}(t) k_{s} / L\left(\mathrm{~W} / \mathrm{m}^{2}\right)$ for instance.

Assume zero initial conditions. Applying the Laplace transform [14, 15] to equation (3), we obtain the following equation

$$
s U(\xi)=\frac{d^{2} U(\xi)}{d \xi^{2}}
$$

where $U(\xi)$ is the Laplace transform of $T_{s}(\xi, \tau)$. The solution of the resulting equation is written as

$$
U=c_{1} e^{\sqrt{s} \xi}+c_{2} e^{-\sqrt{s} \xi}
$$


If we assume the back surface measurements are known, we have the following relationship $[16,18,25]$

$$
\left[\begin{array}{l}
U_{f}(s) \\
Q_{f}(s)
\end{array}\right]=\left[\begin{array}{cc}
\cosh \sqrt{s} & \frac{1}{\sqrt{s}} \sinh \sqrt{s} \\
\sqrt{s} \sinh \sqrt{s} & \cosh \sqrt{s}
\end{array}\right]\left[\begin{array}{l}
U_{b}(s) \\
Q_{b}(s)
\end{array}\right]
$$

where the two vectors represent the Laplace transform of the temperature and heat flux of the front surface and back surface respectively.

The above matrix equation establishes algebraic relationships in the Laplace domain between the temperature and heat flux of the two surfaces. For instance, if the back surface is adiabatic, i.e. $Q_{b}(s)=0$, we have the following relationship between the temperatures on two surfaces:

$$
U_{f}(s)=\cosh \sqrt{s} U_{b}(s),
$$

and the following relationship between the front surface heat flux and the back surface temperature:

$$
Q_{f}(s)=\sqrt{s} \sinh \sqrt{s} U_{b}(s)
$$

To obtain the temperature and heat flux in the time domain from the known solution in the Laplace domain, inverse Laplace transform must be found. The analytical form of the inverse Laplace transform is possible only for a few very special cases $[14,15]$. In the literature, solutions in time domain are obtained through numerical inverse of the transfer function. Because of the convenience of Laplace transform method for various physical problems, there are well over 100 different algorithms available for calculating the inverse Laplace transform [19-24]. In our previous work [18], we have proposed the polynomial approximations to the transfer functions by matching the zeros and poles of the transfer functions. We present a different approach based on the Hadamard Factorization Theorem described in Chapter XI of [24a] in the following.

\section{Infinite product representation of the transfer functions and the iterative solutions in time domain}

Hadamard Factorization Theorem states that if $G(s)$ is a function analytic on the whole complex

plane, satisfying a growth condition $G(s) \leq C e^{|s|^{a}}$ for a positive constant $C$ and $0<a<1$ (or restated in the language of [24a], $G(s)$ is an entire function of order less than one), then $G(s)$ has the representation 


$$
G(s)=G(0) \prod_{k=1}^{\infty}\left(1-\frac{s}{p_{k}}\right)
$$

where $p_{k}$ are the roots of $G(s)$, repeated if necessary in the case of multiple roots. Since $\cosh \sqrt{s}$ and $\sinh \sqrt{s} / \sqrt{s}$ are entire functions of order less than one (specifically we can take $a=1 / 2$ ), it follows that

$$
\cosh \sqrt{s}=\prod_{k=1}^{\infty}\left\{1+\left[\frac{(2 k-1) \pi}{2}\right]^{-2} s\right\}
$$

and

$$
\frac{\sinh \sqrt{s}}{\sqrt{s}}=\prod_{k=1}^{\infty}\left[1+(k \pi)^{-2} s\right]
$$

If finite number of terms are kept in (15) and (16), the resulting polynomials are those obtained in [18]. We have examined the magnitude and phase for $s=i \omega$ of these functions against their polynomial approximations in [18] graphically. In the following, we develop an iterative procedure with which it is easy to implement polynomial approximations to any order. Figure 1 shows the comparison when the polynomials are truncated at $8^{\text {th }}$ order. The exact and approximate curves for magnitude exactly overlap. The difference in phase angles is small but increases at frequency increases. As more terms are included, the phase angle difference is reduced.

With the infinite product representation of the transfer functions, equation (8) can be expressed in the time domain as follows:

$$
T_{f}(\tau)=\prod_{k=1}^{\infty}\left\{1+\left[\frac{(2 k-1) \pi}{2}\right]^{-2} \frac{d}{d \tau}\right\} T_{b}(\tau) .
$$

Equation (17) incorporates no approximations. This compact relationship has not been reported in the literature. Aside from providing a unique perspective on the solutions of the heat equation, it can be used to obtain approximation to the exact solution. The above equation can be written in the iterative form:

$$
T_{f}^{(k+1)}(\tau)=\left\{1+\left[\frac{(2 k+1) \pi}{2}\right]^{-2} \frac{d}{d \tau}\right\} T_{f}^{(k)}(\tau), \text { for } k=1,2, \cdots,
$$

with 


$$
T_{f}^{(1)}(\tau)=\left[1+\left(\frac{\pi}{2}\right)^{-2} \frac{d}{d \tau}\right] T_{b}(\tau)
$$

The exact solution is obtained in the limit:

$$
T_{f}(\tau)=\lim _{k \rightarrow \infty} T_{f}^{(k)}(\tau)
$$

Similarly, equation (11) can be written as

$$
Q_{f}(s)=s \frac{\sinh \sqrt{s}}{\sqrt{s}} U_{b}(s),
$$

which is expressed in time domain as

$$
q_{f}(\tau)=\prod_{k=1}^{\infty}\left[1+(k \pi)^{-2} \frac{d}{d \tau}\right] \frac{d}{d \tau} T_{b}(\tau)
$$

The exact solution for the heat flux can be obtained in the limit:

$$
q_{f}(\tau)=\lim _{k \rightarrow \infty} q_{f}^{(k)}(\tau)
$$

In the above, the sequences are given by the following iterative process:

$$
q_{f}^{(k+1)}(\tau)=\left[1+(k \pi)^{-2} \frac{d}{d \tau}\right] q_{f}^{(k)}(\tau), \text { for } k=1,2, \cdots,
$$

where

$$
q_{f}^{(1)}(\tau)=\frac{d T_{b}(\tau)}{d \tau}
$$

In summary, by expressing the transfer functions as infinite products, we derived the relationships in the time domain. These relationships are further expressed in iterative forms. The iterative forms in (18) and (24) are close to identity when $k$ is large. Therefore, approximate solutions can be achieved when iterations converge.

In practice, the measurements from the back surface are discrete data sampled at a constant rate. In order to apply the iterative process to find the solutions on the front surface, we adopt a method that can be used when only the discrete data are available.

\section{Calculation of the front surface temperature and heat flux from back surface measurement}


Although there is a vast literature in digital signal processing in dealing with discrete data, our solution also requires time derivatives of the sampled data. We have selected the Savitzky-Golay method [2629] for its proven satisfactory performance in handling similar problems. This method is widely used across different disciplines. In fact, the original paper has received well over two thousand citations.

The Savitzky-Golay method performs least-squares fit of a point and $\mathrm{m}$ points on either side of it with a chosen order of polynomial. For points on either edges of the data, asymmetric $2 m+1$ data points are chosen for the fit. This corresponds to performing a moving $2 \mathrm{~m}+1$ least-squares fit across the data. The weights can be easily calculated using Gram polynomials. The weights for a 7 point, quartic polynomial fit are given in the Appendix. As illustrations on using the weights, for given data $y_{1}, y_{2}, \ldots y_{n}$, the smoothed value corresponding to $y_{1}$ is given by

$$
Y_{1}=\frac{1}{462}\left(456 y_{1}+25 y_{2}-35 y_{3}+10 y_{4}+20 y_{5}-19 y_{6}+5 y_{7}\right)
$$

where the weights are given in the second column of the table in the Appendix. The weights in the next two columns are used to calculate $Y_{2}$ and $Y_{3}$ respectively. From $Y_{4}$ to $Y_{n-3}$, i.e. the points away from the edges, the following formula is used:

$$
Y_{i}=\frac{1}{231}\left(5 y_{i-3}-30 y_{i-2}+75 y_{i-1}+131 y_{i}+75 y_{i+1}-30 y_{i+2}+5 y_{i+3}\right)
$$

The derivative calculations are conducted similarly using the weights in the table given in the Appendix.

We apply the methods developed above to calculate the front surface temperature and heat flux while the back surface is assumed to be adiabatic; see Figure 1. Assume that the front surface heat flux is a unit step. The analytical solution is available in the literature [30]:

where

$$
\begin{aligned}
& T_{f}(\tau)=T(\tau, 0) \\
& T_{b}(\tau)=T(\tau, 1)
\end{aligned}
$$

$$
T(\tau, \xi)=\tau+\frac{\xi^{2}}{2}-\xi+\frac{1}{3}-\frac{2}{\pi^{2}} \sum_{\mathrm{n}=1}^{\infty} \frac{\cos (n \pi \xi)}{n^{2}} e^{-(n \pi)^{2} \tau}
$$


Our computation starts from the sampled back surface data $T_{b}\left(\tau_{i}\right)$ given by (29), where $\tau_{i}=0, \delta_{\tau}, 2 \delta_{\tau}, \ldots$ and $\delta_{\tau}$ is the sampling interval. The sampling interval is set to 0.1 in the following. To the sampled exact data, we have added noises as follows:

$$
y_{i}=\left(1+\alpha_{1} n_{1 i}\right) T_{b}\left(\tau_{i}\right)+\alpha_{2} n_{2 i}
$$

where $\alpha_{1}$ and $\alpha_{2}$ are two scaling factors and $n_{1 i}$ and $n_{2 i}$ are pseudorandom numbers following standard normal distribution. Together they simulate sensor noises. The noisy data are fed to Savitzky-Golay algorithm to obtain the smoothing and differentiation of the data and $T_{f}^{(1)}(\tau)$ and $q_{f}^{(1)}(\tau)$ are calculated using (19) and (25). These results are then substituted into (18) and (24) to obtain $T_{f}^{(2)}(\tau)$ and $q_{f}^{(2)}(\tau)$. The iteration is continued until sequences converge.

The two panels in Figure 2 show the reconstructed front surface and temperature from the exact back surface temperature with 8 and 16 iterations respectively. We note that the effect of doubling the number of iterations has a small though noticeable effect. Further doubling of the iteration number generates results which are indistinguishable from those with 16 iterations. Even with only 8 iterations, the front surface temperature agrees with the analytical solution very well. For the front surface heat flux, the agreement is very good except the initial two points. Since the front surface heat flux is discontinuous at $\tau=0$, larger errors occur nearby. The errors at the first two points do not decrease much as the number of iteration is increased further. Other than these two points, the heat flux is very close to the constant input assumed in the analytical solution.

The sampled back surface temperature data are the only data used in the calculation. Sensor noise will unavoidably affect the accuracy of the reconstructed front surface temperature and heat flux. Since the iterative scheme in our approach involves numerical derivatives, noise could potentially get amplified in the process causing the iteration scheme to diverge. In [18], we have found that to recover the front surface heat flux, the noise in the back surface measurement must be so small that it is not feasible using today's sensors. The above iterative procedure uses Savitzky-Golay algorithm for data smoothing and differentiation. The Savitzky-Golay algorithm employs least square fit to the data which has filtering effect. As a result, we found that our iterative procedure can tolerate much larger noise than in [18]. Figure 3 shows the results when noise with $\alpha_{1}=\alpha_{2}=0.01$ is included. Again the data used are sampled temperature marked with symbol ' + '. Deviation of the noisy back surface temperature denoted by '+' from the exact result is noticeable. The top and bottom panels correspond to 8 iterations and 16 iterations respectively. The small differences between the two panels are hardly noticeable.

The two panels in Figure 3 demonstrate that the iterative procedures can tolerate sensor noises. With the sensor noise, the results noticeably deviate from the analytical solutions. The differences in front surface 
heat flux are significant. However, it is not reasonable to regard the differences as errors since the input data (with the sensor noise) no longer correspond to those for the analytical solutions. To further illustrate this point, we show the results in Figure 4 when even large sensor noise, $\alpha_{1}=\alpha_{2}=0.03$, is included. We found that the results change little with the number of iterations. However, they deviate from the analytical solutions even more significantly. The noisy input data are 15 temperature values denoted by '+” symbol in Figure 4 . While $\alpha_{1}=\alpha_{2}=0.03$ may appear to be very small numbers, the corresponding noise is so significant that it has changed the back surface temperature qualitatively. In particular, the temperature at $\tau=0.7$ is lower than at $\tau=0.6$. Since the back surface temperature data are the only input to our iterative procedures, results are obtained accordingly. In other words, deviations from the analytical results corresponding to the constant heat flux are not the correct measures for the accuracy of the procedures. If fact, the results would be suspicious if they show no deviations. Note also that our procedure makes no assumptions about the constant heat flux on the front surface. It is uniquely suited for the transient solutions.

Finally, we consider the implementation of our algorithm when the actual data are the back surface temperature

$$
y_{i}=\left[y_{1}, y_{2}, \cdots, y_{n}\right]
$$

with sampling time interval $\delta t$. We regard the sampled temperature as corresponding to the rescaled time

$$
\tau_{i}=[0, \delta \tau, \cdots,(n-1) \delta \tau],
$$

where

$$
\delta \tau=\frac{\delta t}{t_{c}}
$$

and $t_{c}$ is given in (2). The recovered front surface temperature retains the unit of the back surface measurement. The front surface is given by $q_{f}\left(\tau_{i}\right) k_{s} / L$.

\section{Conclusion}

Based on the Hadamard Factorization Theorem, we developed an iterative procedure to calculate the front surface heat flux and temperature using measurements on the back surface. Combining our procedure with the well-known Savitzky-Golay method for smoothing and differentiation of the sampled data, our procedure is able to tolerate measurement noise. The noise tolerance is a significantly improvement to the work in [18]. Our procedure is not limited to constant heat flux input on the front surface. 


\section{References}

[1] E.M. Sparrow, A. Haji-Sheikh, T.S. Lundgren, The inverse problem in transient heat conduction, ASME J. Appl. Mech. 86 (1964) 369-375.

[2] J.V. Beck, B. Blackwell, and C.R. St-Clair, Inverse Heat Conduction: Ill Posed Problems, Wiley, New York, 1985.

[3] R. Pasquetti, C.L. Niliot, Boundary element approach for inverse heat conduction problems: application to a bidimensional transient numerical experiment, Numer. Heat Transfer, Part B20 (1991) 2911-2919.

[4] O.M. Alifanov, Inverse Heat Transfer Problems, Springer-Verlag, Berlin/Heidelberg, 1994.

[5] C.-Y. Yang, C.-K. Chen, The boundary estimation in two-dimensional inverse heat conduction problems, J. Phys. D: Appl. Phys. 29 (1996) 333-339.

[6] C.-H. Huang, S.-P. Wang, A three-dimensional inverse heat conduction problem in estimating surface heat flux by conjugate gradient method, Int. J. Heat Mass Transfer 42 (1999) 3387-3403.

[7] M.N. Özisik, and H.R.B. Orlande, Inverse Heat Transfer: Fundamentals and Applications, Taylor \& Francis, New York, 2000.

[8] A.F. Emery, A.V. Nenarokomov, T.D. Fadale, Uncertainties in parameter estimation: the optimal experimental design, Int. J. Heat Mass Transfer 43 (2000) 3331-3339.

[9] M. Monde, H. Arima, Y. Mitsutake, Estimation of surface temperature and heat flux using inverse solution for one-dimensional heat conduction, ASME J. Heat Transfer 125 (2003) 213-223.

[10] X. Xue, R. Luck, J.T. Berry, Comparisons and improvements concerning the accuracy and robustness of inverse heat conduction algorithms, Inverse Prob. Sci. Eng. 13 (2) (2005) 177-199.

[11] J. Zhou, Y. Zhang, J.K. Chen, Z.C. Feng, Inverse heat conduction in a finite slab with measured back heat flux, in: Proceedings of the $47^{\text {th }}$ AIAA Aerospace Sciences Meeting, Orlando, FL, January 5-9, 2009.

[12] J. Zhou, Y. Zhang, J.K. Chen, Z.C. Feng, Inverse heat conduction using measured back surface temperature and heat flux, J. Thermophysics and Heat Transfer 24 (2010) 95-103.

[13] J. Zhou, Y. Zhang, J.K. Chen, Z.C. Feng, Inverse heat conduction in a composite slab with pyrolysis effect and temperature-dependent thermophysical properties, ASME J. Heat Transfer 132 (2010) 034502-1.

[14] M.N. Özisik, 1993, Heat Conduction, $2^{\text {nd }}$ ed., Wiley-Interscience, New York.

[15] G.E. Myers, Analytical Methods in Conduction Heat Transfer, $2^{\text {nd }}$ Ed., AMCHT Publications, Madison, WI, 1998.

[16] D. Maillet, S. Andre, J.C. Batsale, A. Degiovanni, C. Moyne, Thermal Quadrupoles: Solving the 
Heat Equation through Integral Transforms, John Wiley \& Sons, New York, 2000.

[17] A. Sutradhar, G.H. Paulino, L.J. Gray, Transient heat conduction in homogeneous and nonhomogeneous materials by the Laplace transform Galerkin boundary element method, Engineering Analysis with Boundary Elements, Vol. 26, 2002, pp. 119-132.

[18] Z.C. Feng, J.K. Chen, and Y. Zhang, Real-time solution of heat conduction in a finite slab for inverse analysis, International Journal of Thermal Sciences, 49 (2010), 762-768.

[19] J. Abate, P.P. Valko, Multi-precision Laplace transform inversion, International Journal for Numerical Methods in Engineering, Vol. 60, 2004, pp. 979-993.

[20] B. Davies and B. Martin, Numerical inversion of the Laplace transform: a survey and comparison of methods. Journal of Computational Physics, Vol. 33, 1979, pp. 1-32.

[21] G.V. Narayanan and D.E. Beskos, Numerical operational methods for time-dependent linear problems. International Journal for Numerical Methods in Engineering, Vol. 18, 1982, pp.1829-1854.

[22] D.G. Duffy, On the numerical inversion of Laplace Transform: comparison of three new methods on characteristic problems from applications. ACM Transactions on Mathematical Software, Vol. 19, 1993, pp. 333-359.

[23] D.Y. Tzou, A Unified Field Theory for Heat Conduction from Macro- to Micro-Scale, ASME Journal of Heat Transfer, Vol. 117 (1995) 8-16.

[24] D.Y. Tzou, Macro- to Microscale Heat Transfer: The Lagging Behavior. Taylor \& Francis, Washington DC, 1997

[24a] J.B. Conway, Functions of One Complex Variable, Second edition. Graduate Texts in Mathematics, Vol. 11, Springer-Verlag, New York - Berlin, 1978.

[25] G.E. Cossali, Dynamic response of a non-homogeneous 1D slab under periodic thermal excitation, International Journal of Heat and Mass Transfer, Vol. 50 (2007), 3943-3948.

[26] A. Savitzky and M.J.E. Golay, Smoothing and differentiation of data by simplified least squares procedures, Anal. Chem. 36 (1964), 1627-1639.

[27] P.A. Goray, General least-squares smoothing and differentiation by the convolution (Savitzky-Golay) method, Anal. Chem. 62 (1990), 570-573.

[28] M. Jakubowska and W.W. Kubiak, Adaptive-degree polynomial filter for voltammetric signal, Analytica Chimica Acta 512 (2004), 241-250.

[29] J. Luo, K. Ying, P. He, and J. Bai, Properties of Savitzky-Golay digital differentiators. Digital Signal Processing, 15 (2005), 122-126.

[30] A. Faghri, Y. Zhang and J.R. Howell, 2010, Advanced Heat and Mass Transfer, Global Digital Press, Columbia, MO. 


\section{Appendix. Tables of weights for seven point, quartic fit}

Convolution weights for quartic smoothing

\begin{tabular}{|c|c|c|c|c|c|c|c|}
\hline $\mathrm{i}$ & -3 & -2 & -1 & 0 & 1 & 2 & 3 \\
\hline-3 & 456 & 25 & -35 & 5 & 20 & -19 & 5 \\
\hline-2 & 25 & 356 & 155 & -30 & -65 & 70 & -19 \\
\hline-1 & -35 & 155 & 212 & 75 & 25 & -65 & 20 \\
\hline 0 & 10 & -60 & 150 & 131 & 150 & -60 & 10 \\
\hline 1 & 20 & -65 & 25 & 75 & 212 & 155 & -35 \\
\hline 2 & -19 & 70 & -65 & -30 & 155 & 356 & 25 \\
\hline 3 & 5 & -19 & 20 & 5 & -35 & 25 & 456 \\
\hline norm & 462 & 462 & 462 & 231 & 462 & 462 & 462 \\
\hline
\end{tabular}

First order derivative:

\begin{tabular}{|c|c|c|c|c|c|c|c|}
\hline $\mathrm{i}$ & -3 & -2 & -1 & 0 & 1 & 2 & 3 \\
\hline-3 & -4420 & -104 & 158 & 22 & -136 & -20 & 746 \\
\hline-2 & 5059 & -25 & -1619 & -67 & 607 & 59 & -2375 \\
\hline-1 & 1504 & 68 & -50 & -58 & -764 & -16 & 1378 \\
\hline 0 & -2394 & 84 & 1218 & 0 & -1218 & -84 & 2394 \\
\hline 1 & -1378 & 16 & 764 & 58 & 50 & -68 & -1504 \\
\hline 2 & 2357 & -59 & -607 & 67 & 1619 & 25 & -5059 \\
\hline 3 & -746 & 20 & 136 & -22 & -158 & 104 & 4420 \\
\hline norm & 2772 & 252 & 2772 & 252 & 2772 & 252 & 2772 \\
\hline
\end{tabular}




\section{Figure Captions}

Figure 1 Comparison of the transfer function in (16) with its polynomial approximations. The three magnitude curves exactly overlap. The difference in phase angles decreases as more terms as included in the product.

Figure 2 One dimensional heat conduction problem with an adiabatic back surface

Figure 3 Comparison of the reconstructed solutions (points) after 16 iterations with the analytical solutions (lines). No noise is included in the back surface temperature, i.e. $\alpha_{1}=\alpha_{2}=0$. Top: 8 iterations; bottom: 16 iterations.

Figure 4 Comparison of the recovered data (points) and the analytical results (lines) with 8 iterations. The symbol '+' represents back surface temperature with noise added. The symbol 'o' represents the calculated front surface temperature and ' $*$ ' represents the calculated front surface heat flux. Calculation is based on data for $\tau=[0,1.5]$. Noise scale factors are $\alpha_{1}=\alpha_{2}=0.01$. Top panel: 8 iterations; bottom panel: 16 iterations.

Figure 5 Comparison of the recovered data (points) and the analytical results (lines) with 16 iterations. The symbol '+' represents back surface temperature with noise added. The symbol 'o' represents the calculated front surface temperature and ' $*$ ' represents the calculated front surface heat flux. Calculation is based on data for $\tau=[0,1.5]$. Noise scale factors are $\alpha_{1}=\alpha_{2}=0.03$;. Top panel: 8 iteration; bottom panel: 16 iteration. 

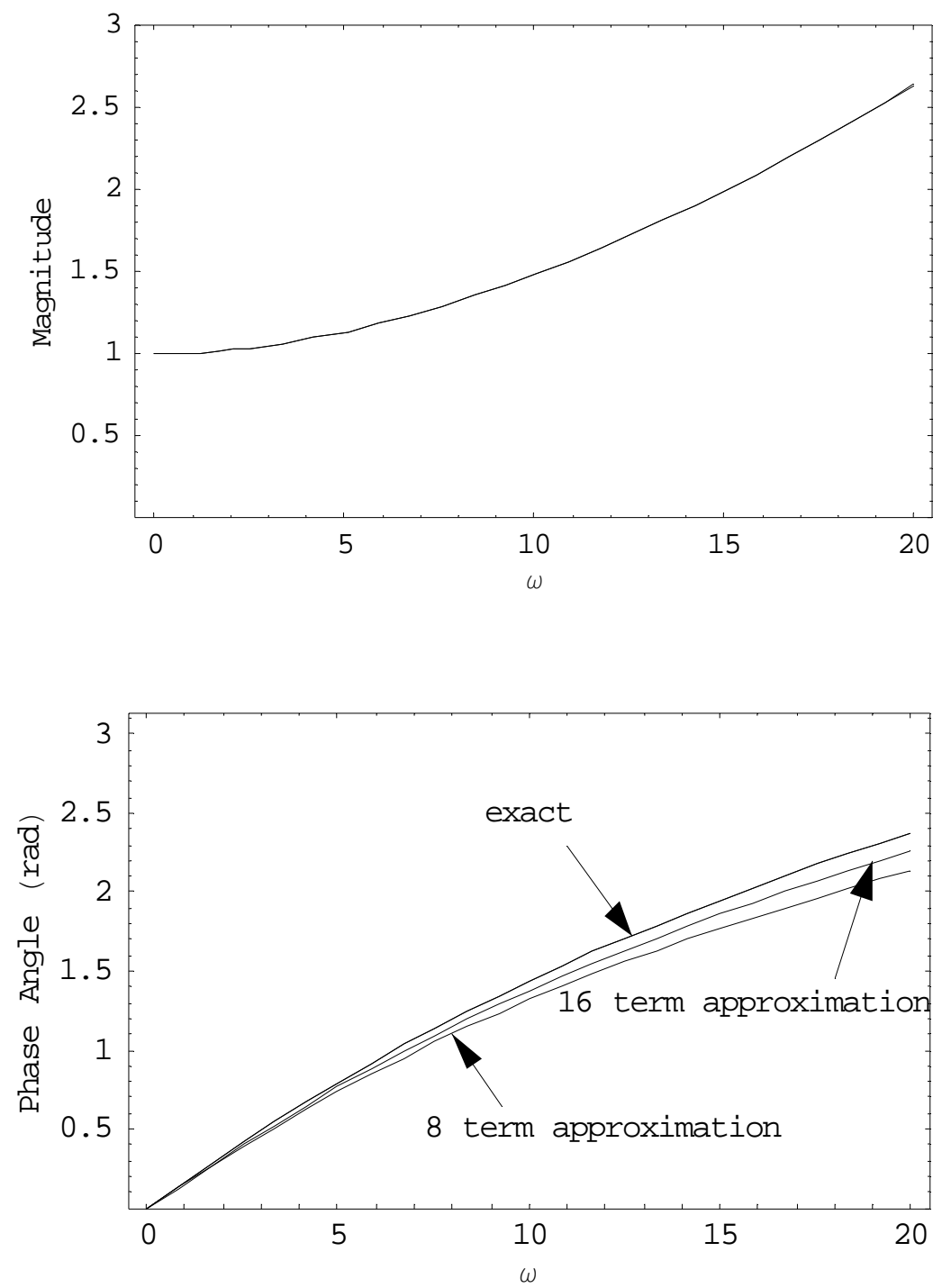

Figure 1 


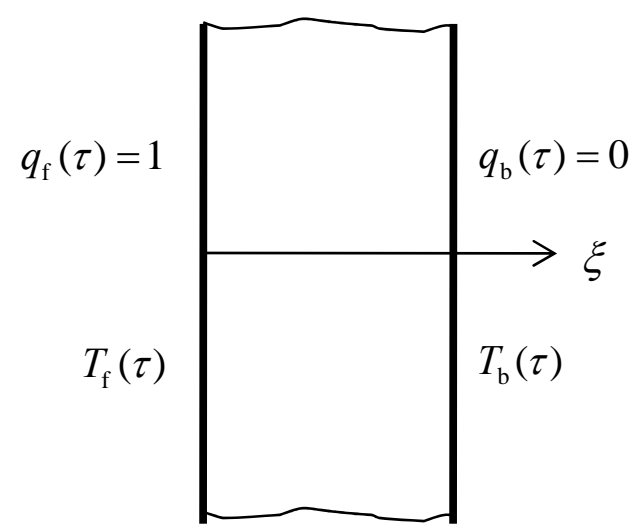

Figure 2 

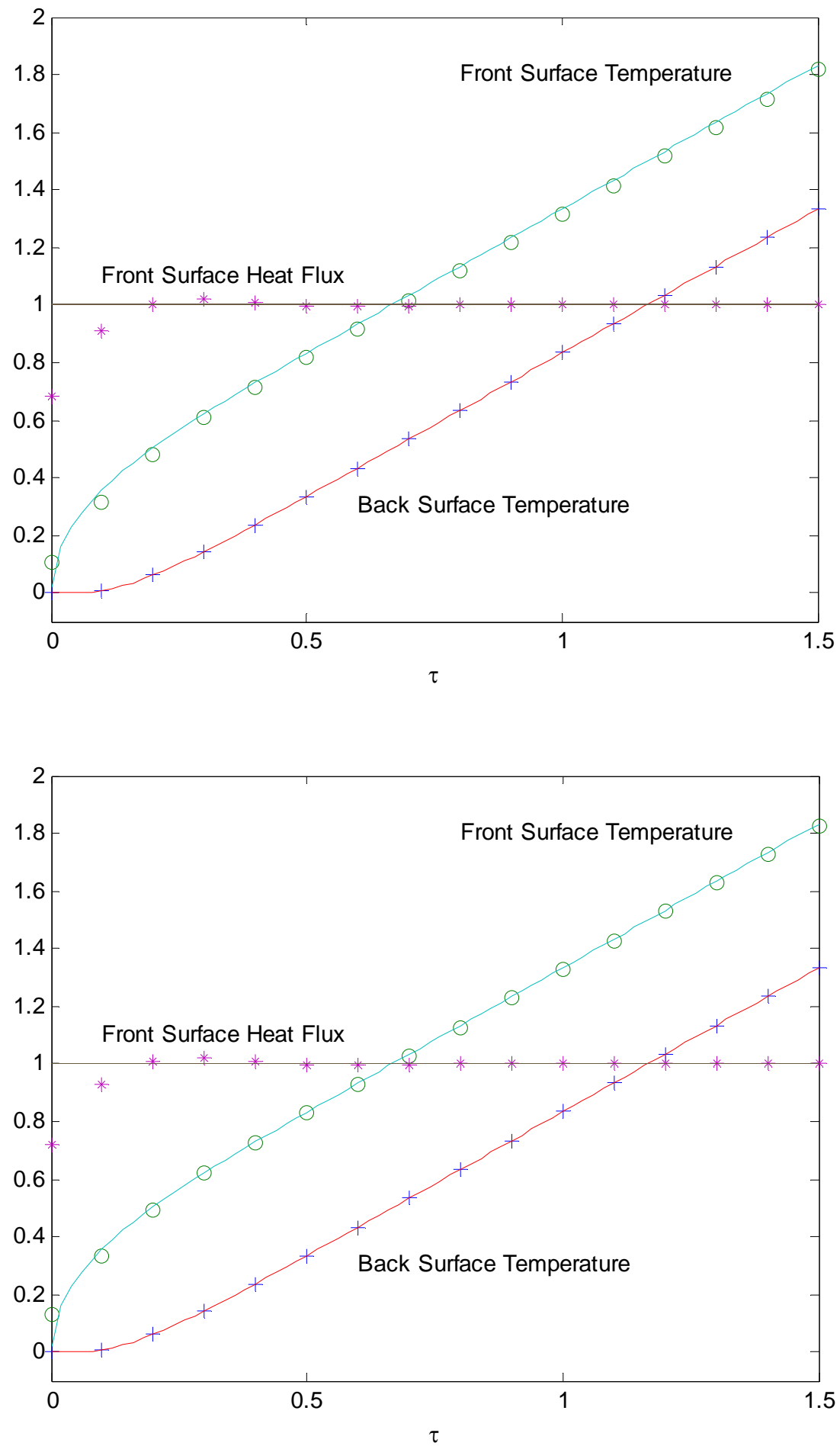

Figure 3 

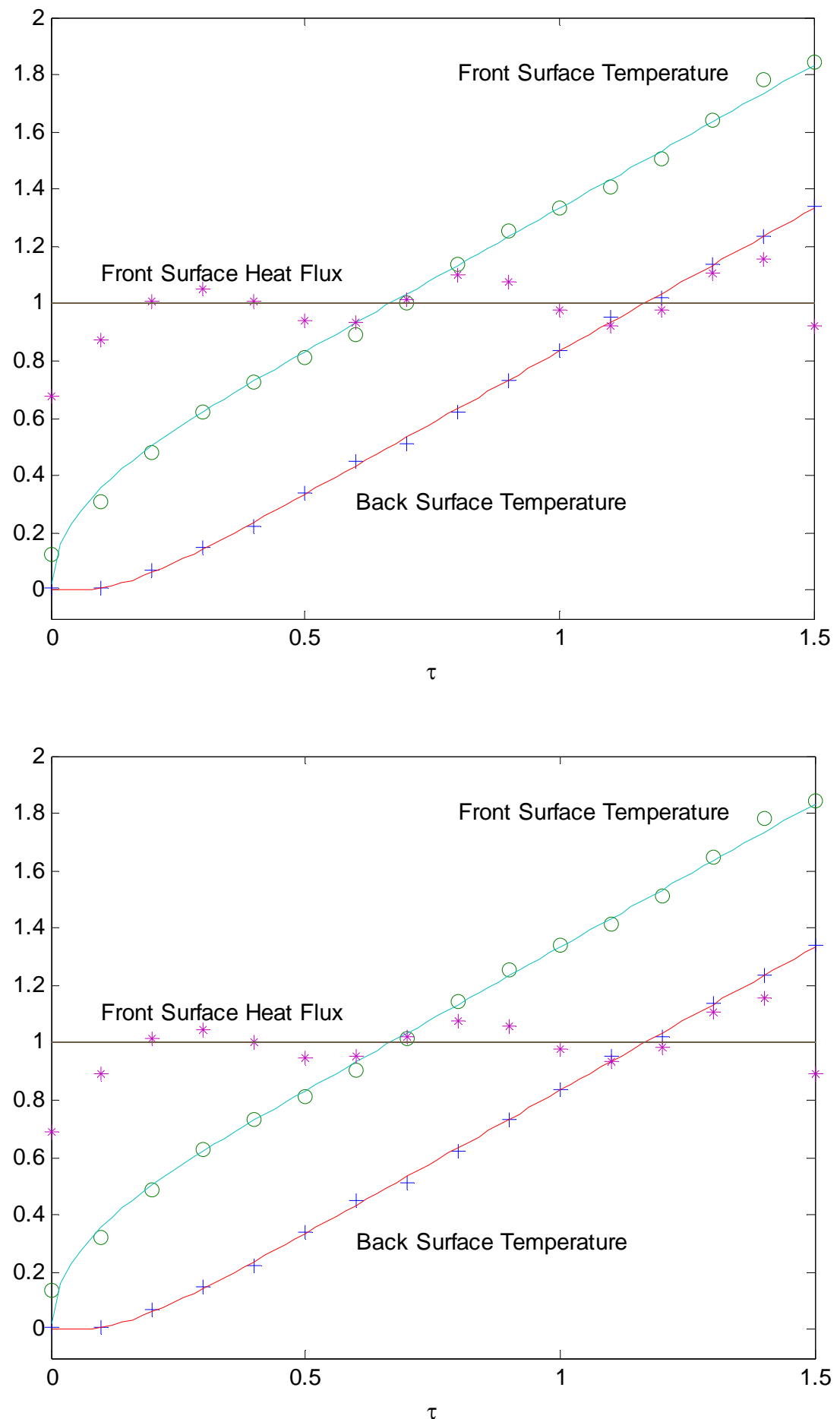

Figure 4 

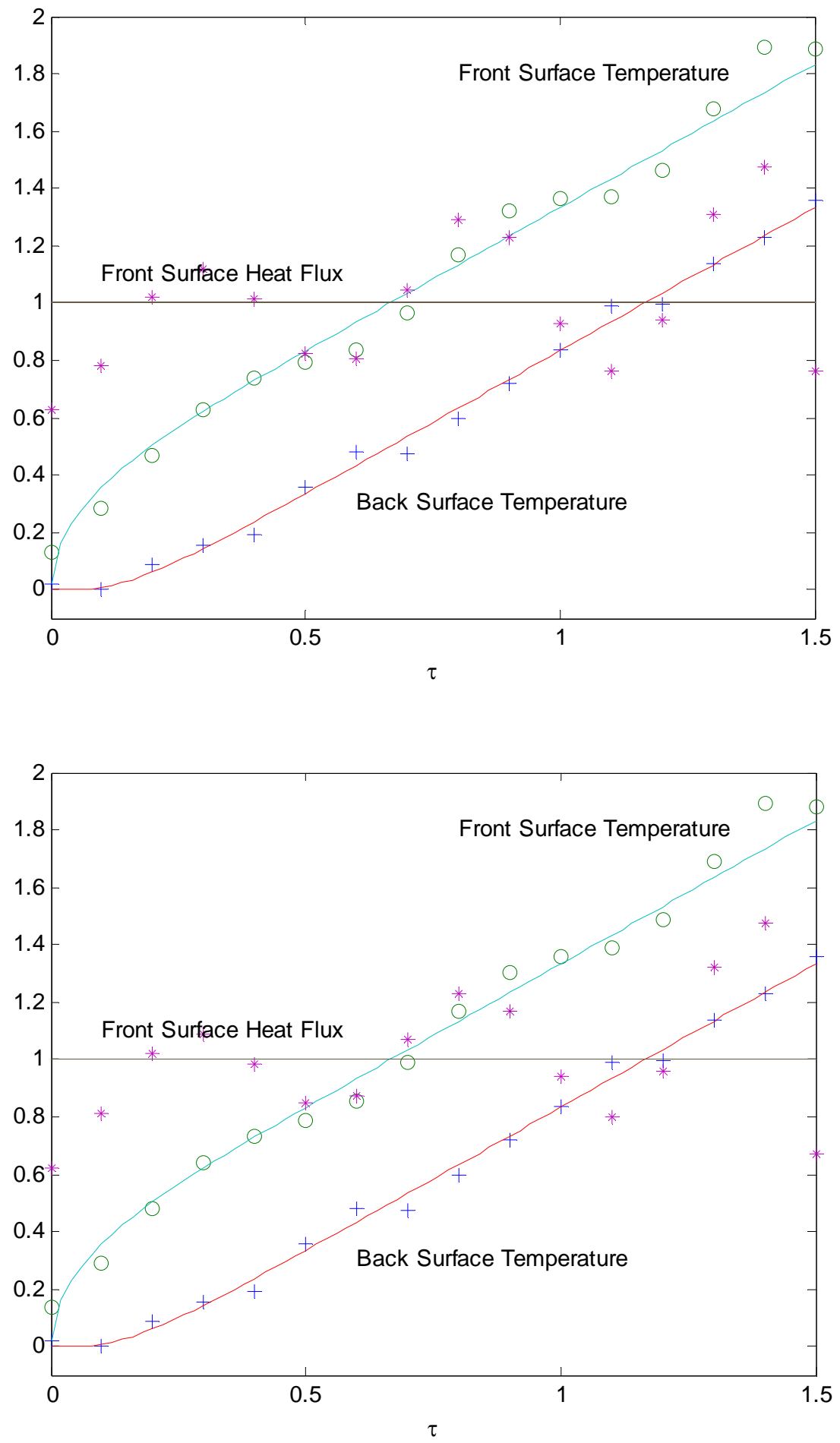

Figure 5 